\title{
Global Attraction to Solitary Waves in Models Based on the Klein-Gordon Equation ${ }^{\star}$
}

\author{
Alexander I. KOMECH ${ }^{\dagger \S}$ and Andrew A. KOMECH ${ }^{\ddagger}$ \\ $\dagger$ Faculty of Mathematics, University of Vienna, Wien A-1090, Austria \\ E-mail: alexander.komech@univie.ac.at \\ URL: http://www . mat. univie.ac.at/ komech/ \\ $\ddagger$ Mathematics Department, Texas A\&M University, College Station, TX 77843, USA \\ E-mail: comech@math.tamu.edu \\ URL: http://www.math.tamu.edu/ comech/ \\ $\S$ Institute for Information Transmission Problems, B. Karetny 19, Moscow 101447, Russia
}

Received November 01, 2007, in final form January 22, 2008; Published online January 31, 2008

Original article is available at http://www.emis.de/journals/SIGMA/2008/010/

\begin{abstract}
We review recent results on global attractors of $\mathbf{U}(1)$-invariant dispersive Hamiltonian systems. We study several models based on the Klein-Gordon equation and sketch the proof that in these models, under certain generic assumptions, the weak global attractor is represented by the set of all solitary waves. In general, the attractors may also contain multifrequency solitary waves; we give examples of systems which contain such solutions.
\end{abstract}

Key words: global attractors; solitary waves; solitary asymptotics; nonlinear Klein-Gordon equation; dispersive Hamiltonian systems; unitary invariance

2000 Mathematics Subject Classification: 35B41; 37K40; 37L30; 37N20; 81Q05

\section{Introduction}

The long time asymptotics for nonlinear wave equations have been the subject of intensive research, starting with the pioneering papers by Segal [44, 45], Strauss [53], and Morawetz and Strauss [39], where the nonlinear scattering and local attraction to zero were considered. Global attraction (for large initial data) to zero may not hold if there are quasistationary solitary wave solutions of the form

$$
\psi(x, t)=\phi(x) e^{-i \omega t}, \quad \text { with } \quad \omega \in \mathbb{R}, \quad \lim _{|x| \rightarrow \infty} \phi(x)=0 .
$$

We will call such solutions solitary waves. Other appropriate names are nonlinear eigenfunctions and quantum stationary states (the solution (1.1) is not exactly stationary, but certain observable quantities, such as the charge and current densities, are time-independent indeed).

Existence of such solitary waves was addressed by Strauss in [54], and then the orbital stability of solitary waves has been studied in [21, 47, 48,49]. The asymptotic stability of solitary waves has been studied by Soffer and Weinstein [50, 51], Buslaev and Perelman [7, 8], and then by others.

The existing results suggest that the set of orbitally stable solitary waves typically forms a local attractor, that is to say, attracts any finite energy solutions that were initially close to it. Moreover, a natural hypothesis is that the set for all solitary waves forms a global attractor of

\footnotetext{
*This paper is a contribution to the Proceedings of the Seventh International Conference "Symmetry in Nonlinear Mathematical Physics" (June 24-30, 2007, Kyiv, Ukraine). The full collection is available at http://www.emis.de/journals/SIGMA/symmetry2007.html
} 
all finite energy solutions. We address this question in the present paper, reviewing the results on the global attraction in several models based on the Klein-Gordon equation, and describing the developed techniques.

We briefly discuss the long-time solitary wave asymptotics for $\mathbf{U}(1)$-invariant Hamiltonian systems in Section 2. The definitions and results on global attraction to solitary waves from the recent papers $[35,36,37]$ are presented in Section 3 . We also give there a very brief sketch of the proof. In Section 4, we give a description of all the steps (omitting excessive technical points) of the argument for the simplest model: Klein-Gordon equation interacting with a nonlinear oscillator. The key parts of the proof are presented in full detail. The examples of (untypical) multifrequency solitary waves are given in Section 5 .

\section{History of solitary asymptotics for U(1)-invariant Hamiltonian systems}

\subsection{Quantum theory}

\section{Bohr's stationary orbits as solitary waves}

Let us focus on the behavior of the electron in the Hydrogen atom. According to Bohr's postulates [4], an unperturbed electron runs forever along certain stationary orbit, which we denote $|E\rangle$ and call quantum stationary state. Once in such a state, the electron has a fixed value of energy $E$, not losing the energy via emitting radiation. The electron can jump from one quantum stationary state to another,

$$
\left|E_{-}\right\rangle \longmapsto\left|E_{+}\right\rangle
$$

emitting or absorbing a quantum of light with the energy equal to the difference of the energies $E_{+}$and $E_{-}$. The old quantum theory was based on the quantization condition

$$
\oint \mathbf{p} \cdot d \mathbf{q}=2 \pi \hbar n, \quad n \in \mathbb{N} .
$$

This condition leads to the values

$$
E_{n}=-\frac{m \mathrm{e}^{4}}{2 \hbar^{2} n^{2}}, \quad n \in \mathbb{N},
$$

for the energy levels in Hydrogen, in a good agreement with the experiment. Apparently, the condition (2.2) did not explain the perpetual circular motion of the electron. According to the classical Electrodynamics, such a motion would be accompanied by the loss of energy via radiation.

In terms of the wavelength $\lambda=\frac{2 \pi \hbar}{|\mathbf{p}|}$ of de Broglie's phase waves [6], the condition (2.2) states that the length of the classical orbit of the electron is the integer multiple of $\lambda$. Following de Broglie's ideas, Schrödinger identified Bohr's stationary orbits, or quantum stationary states $|E\rangle$, with the wave functions that have the form

$$
\psi(\mathbf{x}, t)=\phi_{\omega}(\mathbf{x}) e^{-i \omega t}, \quad \omega=E / \hbar,
$$

where $\hbar$ is Planck's constant. Physically, the charge and current densities

$$
\rho(\mathbf{x}, t)=\mathrm{e} \bar{\psi} \psi, \quad \mathbf{j}(\mathbf{x}, t)=\frac{\mathrm{e}}{2 i}(\bar{\psi} \cdot \nabla \psi-\nabla \bar{\psi} \cdot \psi),
$$

with e $<0$ being the charge of the electron, which correspond to the (quasi)stationary states of the form $\psi(\mathbf{x}, t)=\phi_{\omega}(\mathbf{x}) e^{-i \omega t}$ do not depend on time, and therefore the generated electromagnetic field is also stationary and does not carry the energy away from the system, allowing the electron cloud to flow forever around the nucleus. 


\section{Bohr's transitions as global attraction to solitary waves}

Bohr's second postulate states that the electrons can jump from one quantum stationary state (Bohr's stationary orbit) to another. This postulate suggests the dynamical interpretation of Bohr's transitions as long-time attraction

$$
\Psi(t) \longrightarrow\left|E_{ \pm}\right\rangle, \quad t \rightarrow \pm \infty
$$

for any trajectory $\Psi(t)$ of the corresponding dynamical system, where the limiting states $\left|E_{ \pm}\right\rangle$ generally depend on the trajectory. Then the quantum stationary states $\mathcal{S}_{0}$ should be viewed as the points of the global attractor $\mathscr{A}$.

The attraction (2.3) takes the form of the long-time asymptotics

$$
\psi(x, t) \sim \phi_{\omega_{ \pm}}(x) e^{-i \omega_{ \pm} t}, \quad t \rightarrow \pm \infty,
$$

that hold for each finite energy solution. However, because of the superposition principle, the asymptotics of type (2.4) are generally impossible for the linear autonomous equation, be it the Schrödinger equation

$$
i \hbar \partial_{t} \psi=-\frac{\hbar^{2}}{2 m} \Delta \psi-\frac{\mathrm{e}^{2}}{|\mathbf{x}|} \psi
$$

or relativistic Schrödinger or Dirac equation in the Coulomb field. An adequate description of this process requires to consider the equation for the electron wave function (Schrödinger or Dirac equation) coupled to the Maxwell system which governs the time evolution of the four-potential $A(x, t)=(\varphi(x, t), \mathbf{A}(x, t))$ :

$$
\begin{aligned}
& \left(i \hbar \partial_{t}-\mathrm{e} \varphi\right)^{2} \psi=\left(c \frac{\hbar}{i} \nabla-\mathrm{e} \mathbf{A}\right)^{2} \psi+m^{2} c^{4} \psi, \\
& \square \varphi=4 \pi \mathrm{e}(\bar{\psi} \psi-\delta(\mathbf{x})), \quad \square \mathbf{A}=4 \pi \mathrm{e} \frac{\bar{\psi} \cdot \nabla \psi-\nabla \bar{\psi} \cdot \psi}{2 i} .
\end{aligned}
$$

Consideration of such a system seems inevitable, because, again by Bohr's postulates, the transitions (2.1) are followed by electromagnetic radiation responsible for the atomic spectra. Moreover, the Lamb shift (the energy of $2 S_{1 / 2}$ state being slightly higher than the energy of $2 P_{1 / 2}$ state) can not be explained in terms of the linear Dirac equation in the external Coulomb field. Its theoretical explanation within the Quantum Electrodynamics takes into account the higher order interactions of the electron wave function with the electromagnetic field, referred to as the vacuum polarization and the electron self-energy correction.

The coupled Maxwell-Schrödinger system was initially introduced in [43]. It is a U(1)invariant nonlinear Hamiltonian system. Its global well-posedness was considered in [22]. One might expect the following generalization of asymptotics (2.4) for solutions to the coupled Maxwell-Schrödinger (or Maxwell-Dirac) equations:

$$
(\psi(x, t), A(x, t)) \sim\left(\phi_{\omega_{ \pm}}(x) e^{-i \omega_{ \pm} t}, A_{\omega_{ \pm}}(x)\right), \quad t \rightarrow \pm \infty .
$$

The asymptotics (2.5) would mean that the set of all solitary waves

$$
\left\{\left(\phi_{\omega} e^{-i \omega t}, A_{\omega}\right): \omega \in \mathbb{R}\right\}
$$

forms a global attractor for the coupled system. The asymptotics of this form are not available yet in the context of coupled systems. Let us mention that the existence of the solitary waves for the coupled Maxwell-Dirac equations was established in [16]. 


\subsection{Solitary waves as global attractors for dispersive systems}

Convergence to a global attractor is well known for dissipative systems, like Navier-Stokes equations (see $[1,23,56]$ ). For such systems, the global attractor is formed by the static stationary states, and the corresponding asymptotics (2.4) only hold for $t \rightarrow+\infty$.

We would like to know whether dispersive Hamiltonian systems could, in the same spirit, possess finite dimensional global attractors, and whether such attractors are formed by the solitary waves. Although there is no dissipation per se, we expect that the attraction is caused by certain friction mechanism via the dispersion (local energy decay). Because of the difficulties posed by the system of interacting Maxwell and Dirac (or Schrödinger) fields (and, in particular, absence of the a priori estimates for such systems), we will work with simpler models that share certain key properties of the coupled Maxwell-Dirac or Maxwell-Schrödinger systems. Let us try to single out these key features:

(i) The system is $\mathbf{U}(1)$-invariant.

This invariance leads to the existence of solitary wave solutions $\phi_{\omega}(x) e^{-i \omega t}$.

(ii) The linear part of the system has a dispersive character.

This property provides certain dissipative features in a Hamiltonian system, due to local energy decay via the dispersion mechanism.

(iii) The system is nonlinear.

The nonlinearity is needed for the convergence to a single state of the form $\phi_{\omega}(x) e^{-i \omega t}$. Bohr type transitions to pure eigenstates of the energy operator are impossible in a linear system because of the superposition principle.

We suggest that these are the very features are responsible for the global attraction, such as (2.4), (2.5), to "quantum stationary states".

Remark 1. The global attraction (2.4), (2.5) for $\mathbf{U}(1)$-invariant equations suggests the corresponding extension to general $\mathbf{G}$-invariant equations ( $\mathbf{G}$ being the Lie group):

$$
\psi(x, t) \sim \psi_{ \pm}(x, t)=e^{\boldsymbol{\Omega}_{ \pm} t} \phi_{ \pm}(x), \quad t \rightarrow \pm \infty,
$$

where $\boldsymbol{\Omega}_{ \pm}$belong to the corresponding Lie algebra and $e^{\boldsymbol{\Omega}_{ \pm} t}$ are the one-parameter subgroups. Respectively, the global attractor would consist of the solitary waves (2.6). On a seemingly related note, let us mention that according to Gell-Mann-Ne'eman theory [18] there is a correspondence between the Lie algebras and the classification of the elementary particles which are the "quantum stationary states". The correspondence has been confirmed experimentally by the discovery of the omega-minus Hyperon.

Besides Maxwell-Dirac system, naturally, there are various nonlinear systems under consideration in the Quantum Physics. One of the simpler nonlinear models is the nonlinear KleinGordon equation which takes its origin from the articles by Schiff [41, 42], in his research on the classical nonlinear meson theory of nuclear forces. The mathematical analysis of this equation is started by Jörgens [26] and Segal [44], who studied its global well-posedness in the energy space. Since then, this equation (alongside with the nonlinear Schrödinger equation) has been the main playground for developing tools to handle more general nonlinear Hamiltonian systems. The nonlinear Klein-Gordon equation is a natural candidate for exhibiting solitary asymptotics (2.4).

Now let us describe the existing results on attractors in the context of dispersive Hamiltonian systems. 


\section{Local and global attraction to zero}

The asymptotics of type (2.4) were discovered first with $\psi_{ \pm}=0$ in the scattering theory. Namely, Segal, Morawetz, and Strauss studied the (nonlinear) scattering for solutions of nonlinear KleinGordon equation in $\mathbb{R}^{3}[39,46,53]$. We may interpret these results as local (referring to small initial data) attraction to zero:

$$
\psi(x, t) \sim \psi_{ \pm}=0, \quad t \rightarrow \pm \infty
$$

The asymptotics (2.7) hold on an arbitrary compact set and represent the well-known local energy decay. These results were further extended in [19, 20, 25, 27]. Apparently, there could be no global attraction to zero (global referring to arbitrary initial data) if there are solitary wave solutions $\phi_{\omega}(x) e^{-i \omega t}$.

\section{Solitary waves}

The existence of solitary wave solutions of the form

$$
\psi_{\omega}(x, t)=\phi_{\omega}(x) e^{-i \omega t}, \quad \omega \in \mathbb{R}, \quad \phi_{\omega} \in H^{1}\left(\mathbb{R}^{n}\right),
$$

with $H^{1}\left(\mathbb{R}^{n}\right)$ being the Sobolev space, to the nonlinear Klein-Gordon equation (and nonlinear Schrödinger equation) in $\mathbb{R}^{n}$, in a rather generic situation, was established in [54] (a more general result was obtained in $[2,3]$ ). Typically, such solutions exist for $\omega$ from an interval or a collection of intervals of the real line. We denote the set of all solitary waves by $\mathcal{S}_{0}$.

Due to the $\mathbf{U}(1)$-invariance of the equations, the factor-space $\mathcal{S}_{0} / \mathbf{U}(1)$ in a generic situation is isomorphic to a finite union of intervals. Let us mention that there are numerous results on the existence of solitary wave solutions to nonlinear Hamiltonian systems with $\mathbf{U}(1)$ symmetry. See e.g. $[5,10,17]$.

While all localized stationary solutions to the nonlinear wave equations in spatial dimensions $n \geq 3$ turn out to be unstable (the result known as "Derrick's theorem" [15]), quasistationary solitary waves can be orbitally stable. Stability of solitary waves takes its origin from [58] and has been extensively studied by Strauss and his school in [21, 47, 48, 49].

\section{Local attraction to solitary waves}

First results on the asymptotics of type (2.4) with $\omega_{ \pm} \neq 0$ were obtained for nonlinear $\mathbf{U}(1)$ invariant Schrödinger equations in the context of asymptotic stability. This establishes asymptotics of type (2.4) but only for solutions close to the solitary waves, proving the existence of a local attractor. This was first done by Soffer and Weinstein and by Buslaev and Perelman in $[7,8,50,51]$, and then developed in $[9,12,13,14,40,52]$ and other papers.

\section{Global attraction to solitary waves}

The global attraction of type (2.4) with $\psi_{ \pm} \neq 0$ and $\omega_{ \pm}=0$ was established in certain models in $[28,29,30,31,32,33]$ for a number of nonlinear wave problems. There the attractor is the set of all static stationary states. Let us mention that this set could be infinite and contain continuous components.

In [34] and [35], the attraction to the set of solitary waves (see Fig. 1) is proved for the Klein-Gordon field coupled to a nonlinear oscillator. In [36], this result has been generalized for the Klein-Gordon field coupled to several oscillators. The paper [37] gives the extension to the higher-dimensional setting for a model with the nonlinear self-interaction of the mean field type. We are going to describe these results in this survey. 


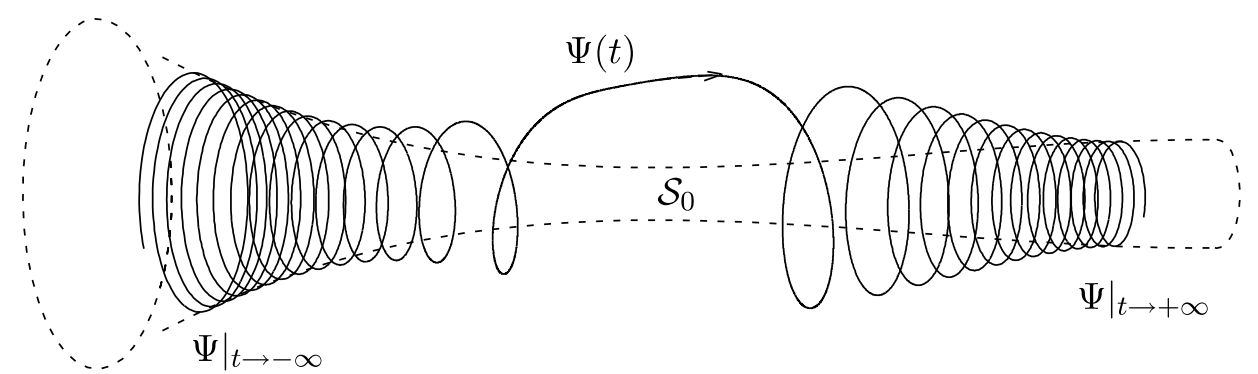

Figure 1. For $t \rightarrow \pm \infty$, a finite energy solution $\Psi(t)$ approaches the global attractor $\mathscr{A}$ which coincides with the set of all solitary waves $\mathcal{S}_{0}$.

We are aware of but one recent advance [55] in the field of nontrivial (nonzero) global attractors for Hamiltonian PDEs. In that paper, the global attraction for the nonlinear Schrödinger equation in dimensions $n \geq 5$ was considered. The dispersive (outgoing) wave was explicitly specified using the rapid decay of local energy in higher dimensions. The global attractor was proved to be compact, but it was neither identified with the set of solitary waves nor was proved to be finite-dimensional [55, Remark 1.18].

\section{Assumptions and results}

In $[35,36,37]$ we introduce the models which possess the key properties we mentioned above: $\mathbf{U}(1)$-invariance, dispersive character, and the nonlinearity. The models allow to prove the global attraction to solitary waves and to develop certain techniques which we hope will allow us to approach more general systems.

\section{Model 1: Klein-Gordon field with a nonlinear oscillator}

We consider the Cauchy problem for the Klein-Gordon equation with the nonlinearity concentrated at the origin:

$$
\begin{aligned}
& \ddot{\psi}(x, t)=\psi^{\prime \prime}(x, t)-m^{2} \psi(x, t)+\delta(x) F(\psi(0, t)), \quad x \in \mathbb{R}, \\
& \left.\psi\right|_{t=0}=\psi_{0}(x),\left.\quad \dot{\psi}\right|_{t=0}=\pi_{0}(x) .
\end{aligned}
$$

Above, $m>0$ and $F$ is a function describing an oscillator at the point $x=0$. The dots stand for the derivatives in $t$, and the primes for the derivatives in $x$. All derivatives and the equation are understood in the sense of distributions. We assume that equation (3.1) is $\mathbf{U}(1)$-invariant; that is,

$$
F\left(e^{i \theta} \psi\right)=e^{i \theta} F(\psi), \quad \theta \in \mathbb{R}, \quad \psi \in \mathbb{C} .
$$

If we identify a complex number $\psi=u+i v \in \mathbb{C}$ with the two-dimensional vector $(u, v) \in \mathbb{R}^{2}$, then, physically, equation (3.1) describes small crosswise oscillations of the infinite string in three-dimensional space $(x, u, v)$ stretched along the $x$-axis. The string is subject to the action of an "elastic force" $-m^{2} \psi(x, t)$ and coupled to a nonlinear oscillator of force $F(\psi)$ attached at the point $x=0$. We assume that the oscillator force $F$ admits a real-valued potential,

$$
F(\psi)=-\nabla_{\psi} U(\psi), \quad \psi \in \mathbb{C}, \quad U \in C^{2}(\mathbb{C}),
$$

where the gradient is taken with respect to $\operatorname{Re} \psi$ and $\operatorname{Im} \psi$.

Remark 2. Viewing the model as an infinite string in $\mathbb{R}^{3}$, the assumption (3.2) means that the potential $U(\psi)$ is rotation-invariant with respect to the $x$-axis. 
The codes for the numerical simulation of a finite portion of a string coupled to an oscillator, with transparent boundary conditions $\left(\partial_{t} \psi= \pm \partial_{x} \psi\right)$, are available in [11].

\section{Model 2: Klein-Gordon field with several nonlinear oscillators}

More generally, we consider the Cauchy problem for the Klein-Gordon equation with the nonlinearity concentrated at the points $X_{1}<X_{2}<\cdots<X_{N}$ :

$$
\begin{aligned}
& \ddot{\psi}(x, t)=\psi^{\prime \prime}(x, t)-m^{2} \psi(x, t)+\sum_{J=1}^{N} \delta\left(x-X_{J}\right) F_{J}\left(\psi\left(X_{J}, t\right)\right), \quad x \in \mathbb{R}, \\
& \left.\psi\right|_{t=0}=\psi_{0}(x),\left.\quad \dot{\psi}\right|_{t=0}=\pi_{0}(x) .
\end{aligned}
$$

\section{Model 3: Klein-Gordon field with the mean field interaction}

We also consider the Klein-Gordon equation with the mean field interaction:

$$
\begin{aligned}
& \ddot{\psi}(x, t)=\Delta \psi(x, t)-m^{2} \psi(x, t)+\rho(x) F(\langle\rho, \psi(\cdot, t)\rangle), \quad x \in \mathbb{R}^{n}, \quad n \geq 1, \\
& \left.\psi\right|_{t=0}=\psi_{0}(x),\left.\quad \dot{\psi}\right|_{t=0}=\pi_{0}(x) .
\end{aligned}
$$

Above, $\rho$ is a smooth real-valued coupling function from the Schwartz class: $\rho \in \mathscr{S}\left(\mathbb{R}^{n}\right), \rho \not \equiv 0$, and

$$
\langle\rho, \psi(\cdot, t)\rangle=\int_{\mathbb{R}^{n}} \rho(x) \psi(x, t) d x .
$$

\section{Hamiltonian structure}

Equations (3.3), (3.4) formally can be written as a Hamiltonian system,

$$
\dot{\Psi}(t)=\mathcal{J} D \mathcal{H}(\Psi), \quad \mathcal{J}=\left[\begin{array}{cc}
0 & 1 \\
-1 & 0
\end{array}\right]
$$

where $\Psi=(\psi, \pi)$ and $D \mathcal{H}$ is the Fréchet derivative of the Hamilton functionals

$$
\begin{aligned}
& \mathcal{H}_{\mathrm{osc}}(\psi, \pi)=\frac{1}{2} \int_{\mathbb{R}}\left(|\pi|^{2}+\left|\psi^{\prime}\right|^{2}+m^{2}|\psi|^{2}\right) d x+\sum_{J} U_{J}\left(\psi\left(X_{J}\right)\right), \\
& \mathcal{H}_{\text {m.f. }}(\psi, \pi)=\frac{1}{2} \int_{\mathbb{R}^{n}}\left(|\pi|^{2}+|\nabla \psi|^{2}+m^{2}|\psi|^{2}\right) d x+U(\langle\rho, \psi\rangle) .
\end{aligned}
$$

Since (3.3) and (3.4) are $\mathbf{U}(1)$-invariant, the Nöther theorem formally implies that the value of the charge functional

$$
\mathcal{Q}(\psi, \pi)=\frac{i}{2} \int(\bar{\psi} \pi-\bar{\pi} \psi) d x
$$

is conserved for solutions $\Psi(t)=(\psi(t), \pi(t))$ to $(3.5)$.

Let us introduce the phase space $\mathscr{E}$ of finite energy states for equations (3.3), (3.4). Denote by $\|\cdot\|_{L^{2}}$ the norm in the complex Hilbert space $L^{2}\left(\mathbb{R}^{n}\right)$ and by $\|\cdot\|_{L_{R}^{2}}$ the norm in $L^{2}\left(\mathbb{B}_{R}^{n}\right)$, where $\mathbb{B}_{R}^{n}$ is a ball of radius $R>0$. Denote by $\|\cdot\|_{H_{R}^{s}}$ the norm in the Sobolev space $H^{s}\left(\mathbb{B}_{R}^{n}\right)$ (which is the dual to the Sobolev space $H_{0}^{-s}\left(\mathbb{B}_{R}^{n}\right.$ ) of functions supported in the ball of radius $R$ ). 


\section{Definition 1 (The phase space).}

(i) $\mathscr{E}=H^{1}\left(\mathbb{R}^{n}\right) \oplus L^{2}\left(\mathbb{R}^{n}\right), n \geq 1$, is the Hilbert space of the states $(\psi, \pi)$, with the norm

$$
\|(\psi, \pi)\|_{\mathscr{E}}^{2}:=\|\pi\|_{L^{2}}^{2}+\|\nabla \psi\|_{L^{2}}^{2}+m^{2}\|\psi\|_{L^{2}}^{2}
$$

(ii) For $\epsilon \geq 0, \mathscr{E}^{-\epsilon}=H^{1-\epsilon}\left(\mathbb{R}^{n}\right) \oplus H^{-\epsilon}\left(\mathbb{R}^{n}\right)$ is the space with the norm

$$
\|(\psi, \pi)\|_{\mathscr{E}-\epsilon}=\left\|(1-\Delta)^{-\epsilon / 2}(\psi, \pi)\right\|_{\mathscr{E}} .
$$

(iii) Define the seminorms

$$
\|(\psi, \pi)\|_{\mathscr{E}-\epsilon, R}^{2}:=\|\pi\|_{H_{R}^{-\epsilon}}^{2}+\|\nabla \psi\|_{H_{R}^{-\epsilon}}^{2}+m^{2}\|\psi\|_{H_{R}^{-\epsilon}}^{2}, \quad R>0 .
$$

$\mathscr{E}_{F}^{-\epsilon}$ is the space with the norm

$$
\|(\psi, \pi)\|_{\mathscr{E}_{F}^{-\epsilon}}=\sum_{R=1}^{\infty} 2^{-R}\|(\psi, \pi)\|_{\mathscr{E}-\epsilon, R}
$$

Equations (3.3), (3.4) are formally Hamiltonian systems with the Hamilton functionals $\mathcal{H}_{\text {osc }}$ and $\mathcal{H}_{\text {m.f. }}$, respectively, and with the phase space $\mathscr{E}$ from Definition 1 (for equation (3.3), the dimension is $n=1$ ). Both $\mathcal{H}_{\text {osc }}$ (or $\mathcal{H}_{\text {m.f. }}$ ) and $\mathcal{Q}$ are continuous functionals on $\mathscr{E}$.

\section{Global well-posedness}

Theorem 1 (Global well-posedness). Assume that the nonlinearity in (3.3) is given by $F_{J}(z)=-\nabla U_{J}(z)$ with $\inf _{z \in \mathbb{C}} U_{J}(z)>-\infty, 1 \leq J \leq N \quad$ or $F(z)=-\nabla U(z)$ with $\inf _{z \in \mathbb{C}} U(z)>-\infty$ in (3.4), respectively). Then:

(i) For every $\left(\psi_{0}, \pi_{0}\right) \in \mathscr{E}$ the Cauchy problem (3.3) ((3.4), respectively) has a unique global solution $\psi(t)$ such that $(\psi, \dot{\psi}) \in C(\mathbb{R}, \mathscr{E})$.

(ii) The map $W(t):\left(\psi_{0}, \pi_{0}\right) \mapsto(\psi(t), \dot{\psi}(t))$ is continuous in $\mathscr{E}$ for each $t \in \mathbb{R}$.

(iii) The energy and charge are conserved: $\mathcal{H}(\psi(t), \dot{\psi}(t))=$ const, $\mathcal{Q}(\psi(t), \dot{\psi}(t))=$ const, $t \in \mathbb{R}$.

(iv) The following a priori bound holds:

$$
\|(\psi(t), \dot{\psi}(t))\|_{\mathscr{E}} \leq C\left(\psi_{0}, \pi_{0}\right), \quad t \in \mathbb{R}
$$

(v) For any $\epsilon \in[0,1]$,

$$
(\psi, \dot{\psi}) \in C^{(\epsilon)}\left(\mathbb{R}, \mathscr{E}^{-\epsilon}\right)
$$

where $C^{(\epsilon)}$ denotes the space of Hölder-continuous functions.

The proof is contained in $[35,36]$, and [37]. 


\section{Solitary waves}

\section{Definition 2 (Solitary waves).}

(i) The solitary waves of equation (3.3) are solutions of the form

$$
\psi(x, t)=\phi_{\omega}(x) e^{-i \omega t}, \quad \text { where } \quad \omega \in \mathbb{R}, \quad \phi_{\omega} \in H^{1}\left(\mathbb{R}^{n}\right) .
$$

(ii) The set of all solitary waves is $\mathcal{S}_{0}=\left\{\phi_{\omega}: \omega \in \mathbb{R}, \phi_{\omega} \in H^{1}\left(\mathbb{R}^{n}\right)\right\}$.

(iii) The solitary manifold is the set $\mathcal{S}=\left\{\left(\phi_{\omega},-i \omega \phi_{\omega}\right): \omega \in \mathbb{R}, \phi_{\omega} \in H^{1}\left(\mathbb{R}^{n}\right)\right\} \subset \mathscr{E}$.

\section{Remark 3.}

(i) The $\mathbf{U}(1)$ invariance of (3.3) and (3.4) implies that the sets $\mathcal{S}_{0}, \mathcal{S}$ are invariant under multiplication by $e^{i \theta}, \theta \in \mathbb{R}$.

(ii) Let us note that for any $\omega \in \mathbb{R}$ there is a zero solitary wave with $\phi_{\omega}(x) \equiv 0$ since $F_{J}(0)=0$.

The following proposition provides a concise description of all solitary wave solutions to (3.1).

Proposition 1. There are no nonzero solitary waves for $|\omega| \geq m$.

For a particular $\omega \in(-m, m)$, there is a nonzero solitary wave solution to (3.1) if and only if there exists $C \in \mathbb{C} \backslash\{0\}$ so that

$$
2 \kappa(\omega)=F(C) / C
$$

The solitary wave solution is given by

$$
\phi_{\omega}(x)=C e^{-\kappa(\omega)|x|}, \quad \kappa(\omega)=\sqrt{m^{2}-\omega^{2}} .
$$

Remark 4. There could be more than one value $C>0$ satisfying (3.9).

Remark 5. By (3.10), $\omega= \pm m$ can not correspond to a nonzero solitary wave.

Proof. When we substitute the ansatz $\phi_{\omega} e^{-i \omega t}$ into (3.1), we get the following relation:

$$
-\omega^{2} \phi_{\omega}(x)=\phi_{\omega}^{\prime \prime}(x)-m^{2} \phi_{\omega}(x)+\delta(x) F\left(\phi_{\omega}(x)\right), \quad x \in \mathbb{R} .
$$

The phase factor $e^{-i \omega t}$ has been canceled out. Equation (3.11) implies that away from the origin we have

$$
\phi_{\omega}^{\prime \prime}(x)=\left(m^{2}-\omega^{2}\right) \phi_{\omega}(x), \quad x \neq 0,
$$

hence $\phi_{\omega}(x)=C_{ \pm} e^{-\kappa_{ \pm}|x|}$ for $\pm x>0$, where $\kappa_{ \pm}$satisfy $\kappa_{ \pm}^{2}=m^{2}-\omega^{2}$. Since $\phi_{\omega}(x) \in H^{1}$, it is imperative that $\kappa_{ \pm}>0$; we conclude that $|\omega|<m$ and that $\kappa_{ \pm}=\sqrt{m^{2}-\omega^{2}}>0$. Moreover, since the function $\phi_{\omega}(x)$ is continuous, $C_{-}=C_{+}=C \neq 0$ (since we are looking for nonzero solitary waves). We see that

$$
\phi_{\omega}(x)=C e^{-\kappa|x|}, \quad C \neq 0, \quad \kappa \equiv \sqrt{m^{2}-\omega^{2}}>0 .
$$

Equation (3.11) implies the following jump condition at $x=0$ :

$$
0=\phi_{\omega}^{\prime}(0+)-\phi_{\omega}^{\prime}(0-)+F\left(\phi_{\omega}(0)\right),
$$

which is satisfied due to (3.9) and (3.12). 


\section{Global attraction to solitary waves}

We will combine the results for the Klein-Gordon equation with one and several oscillators ((3.1) and (3.3)).

Theorem 2 (Global attraction for (3.3), Klein-Gordon equation with $N$ oscillators). Assume that all the oscillators are strictly nonlinear: for all $1 \leq J \leq N$,

$$
\begin{aligned}
& F_{J}(\psi)=-\nabla U_{J}(\psi), \\
& \text { where } \quad U_{J}(\psi)=\sum_{l=0}^{p_{J}} u_{J, l}|\psi|^{2 l}, \quad u_{J, l} \in \mathbb{R}, \quad u_{J, p_{J}}>0, \quad \text { and } \quad p_{J} \geq 2 .
\end{aligned}
$$

Further, if $N \geq 2$, assume that the intervals $\left[X_{J}, X_{J+1}\right], 1 \leq J \leq N-1$, are small enough so that

$$
\min _{1 \leq J \leq N-1}\left(\frac{\pi^{2}}{\left|X_{J+1}-X_{J}\right|^{2}}+m^{2}\right)^{1 / 2}>m \max _{1 \leq J \leq N} \min \left(\prod_{l=1}^{J}\left(2 p_{l}-1\right), \prod_{l=J}^{N}\left(2 p_{l}-1\right)\right),
$$

where $p_{J}$ are exponentials from (3.13). Then for any $\left(\psi_{0}, \pi_{0}\right) \in \mathscr{E}$ the solution $\psi(t)$ to the Cauchy problem (3.3) converges to $\mathcal{S}$ :

$$
\lim _{t \rightarrow \pm \infty} \operatorname{dist}_{\mathscr{E}_{F}}((\psi(t), \dot{\psi}(t)), \mathcal{S})=0
$$

Theorem 3 (Global attraction for (3.4), Klein-Gordon equation with mean field interaction). Assume that the nonlinearity $F(z)$ is strictly nonlinear:

$$
F(z)=-\nabla U(z), \quad \text { where } \quad U(z)=\sum_{l=0}^{p} u_{l}|z|^{2 l}, \quad u_{l} \in \mathbb{R}, \quad u_{p}>0, \quad \text { and } \quad p \geq 2 .
$$

Further, assume that the set

$$
Z_{\rho}=\left\{\omega \in \mathbb{R} \backslash[-m, m]: \hat{\rho}(\xi)=0 \text { for all } \xi \in \mathbb{R}^{n} \text { such that } m^{2}+\xi^{2}=\omega^{2}\right\}
$$

is finite and that

$$
\sigma(\omega):=\frac{1}{(2 \pi)^{n}} \int_{\mathbb{R}^{n}} \frac{|\hat{\rho}(\xi)|^{2}}{\xi^{2}+m^{2}-\omega^{2}} d^{n} \xi
$$

does not vanish at the points $\omega \in Z_{\rho}$. Then for any $\left(\psi_{0}, \pi_{0}\right) \in \mathscr{E}$ the solution $\psi(t) \in C(\mathbb{R}, \mathscr{E})$ to the Cauchy problem (3.4) converges to $\mathcal{S}$ in the space $\mathscr{E}_{F}^{-\epsilon}$, for any $\epsilon>0$ :

$$
\lim _{t \rightarrow \pm \infty} \operatorname{dist}_{\mathscr{E}_{F}^{-\epsilon}}(\Psi(t), \mathcal{S})=0 .
$$

Above, $\operatorname{dist}_{\mathscr{E}_{F}^{-\epsilon}}(\Psi, \mathcal{S}):=\inf _{\Phi \in \mathcal{S}}\|\Psi-\Phi\|_{\mathscr{E}_{F}^{-\epsilon}}$, with $\|\cdot\|_{\mathscr{E}_{F}^{-\epsilon}}$ is defined in (3.6).

Theorem 2 is proved in [36]; Theorem 3 is proved in [37]. We present the sketch of the proof of Theorem 2 for one oscillator in Section 4.

Let us mention several important points.

(i) In the linear case, the global attractor contains the linear span of points of the solitary manifold, $\langle\mathcal{S}\rangle$. In [35], we prove that for the model of one linear oscillator attached to the Klein-Gordon field the global attractor indeed coincides with $\langle\mathcal{S}\rangle$. 
(ii) The condition (3.14) allows to avoid "trapped modes", which could also be characterized as multifrequency solitary waves. In Proposition 8 below, we give an example of such solutions in the situation when the condition (3.14) is violated.

Similarly, the condition of Theorem 3 that $\sigma(\omega)$ does not vanish for $\omega \in Z_{\rho}$ allows to avoid multifrequency solitary waves.

(iii) We prove the attraction of any finite energy solution to the solitary manifold $\mathcal{S}$ :

$$
(\psi(t), \dot{\psi}(t)) \longrightarrow \mathcal{S}, \quad t \rightarrow \pm \infty
$$

where the convergence holds in local seminorms. In this sense, $\mathcal{S}$ is a weak (convergence is local in space) global (convergence holds for arbitrary initial data) attractor.

(iv) $\mathcal{S}$ can be at most a weak attractor because we need to keep forgetting about the outgoing dispersive waves, so that the dispersion plays the role of friction. A strong attractor would have to consist of the direct sum of $\mathcal{S}$ and the space of outgoing waves.

$(v)$ We interpret the local energy decay caused by dispersion as a certain friction effect in order to clarify the cause of the convergence to the attractor in a Hamiltonian model. This "friction" does not contradict the time reversibility: if the system develops backwards in time, one observes the same local energy decay which leads to the convergence to the attractor as $t \rightarrow-\infty$.

(vi) Although we proved the attraction (3.17) to $\mathcal{S}$, we have not proved the attraction to a particular solitary wave, falling short of proving (2.4). Hypothetically, if $\mathcal{S} / \mathbf{U}(1)$ contains continuous components, a solution can be drifting along $\mathcal{S}$, keeping asymptotically close to it, but never approaching a particular solitary wave. This could be viewed as the adiabatic modulation of solitary wave parameters. Apparently, if $\mathcal{S} / \mathbf{U}(1)$ is discrete, a solution converges to a particular solitary wave.

(vii) The requirement that the nonlinearity is polynomial allows us to apply the Titchmarsh convolution theorem. This step is vital in our approach. We do not know whether the polynomiality requirement could be dropped.

(viii) For the real initial data, we obtain a real-valued solution $\psi(t)$. Therefore, the convergence $(3.15),(3.16)$ of $\Psi(t)=(\psi(t), \dot{\psi}(t))$ to the set of pairs $\left(\phi_{\omega},-i \omega \phi_{\omega}\right)$ with $\omega \in \mathbb{R} \backslash\{0\}$ implies that $\psi(t)$ locally converges to zero.

\section{Sketch of the proof}

First, we introduce a concept of the omega-limit trajectory $\beta(x, t)$ which plays a crucial role in the proof.

Definition 3 (Omega-limit trajectory). The function $\beta(x, t)$ is an omega-limit trajectory if there is a global solution $\psi \in C(\mathbb{R}, \mathscr{E})$ and a sequence of times $\left\{s_{j}: j \in \mathbb{N}\right\}$ with $\lim _{j \rightarrow \infty} s_{j}=\infty$ so that

$$
\psi\left(x, t+s_{j}\right) \rightarrow \beta(x, t),
$$

where the convergence is in $C_{b}\left([-T, T] \times \mathbb{B}_{R}^{n}\right)$ for any $T>0$ and $R>0$.

We are going to prove that all omega-limit trajectories are solitary waves: $\beta(x, t)=\phi_{\omega}(x) e^{-i \omega t}$. It suffices to prove that the time spectrum of any omega-limit trajectory $\beta$ consists of at most one frequency.

To complete this program, we study the time spectrum of solutions, that is, their complex Fourier-Laplace transform in time. First, we prove that the spectral density of a solution is 
absolutely continuous for $|\omega|>m$ hence the corresponding component of the solution disperses completely. It follows that the time-spectrum of omega-limit trajectory $\beta$ is contained in a finite interval $[-m, m]$.

Second, we notice that $\beta$ also satisfies the original nonlinear equation. Since the spectral support of $\beta$ is compact and the nonlinearity is polynomial, we may apply the Titchmarsh convolution theorem. This theorem allows to conclude that the spectral support of the nonlinearity would be strictly larger than the spectral support of the linear terms in the equation (which would be a contradiction!) except in the case when the spectrum of the omega-limit trajectory consists of a single frequency $\omega_{+} \in[-m, m]$.

Since any omega-limit trajectory is a solitary wave, the attraction (3.17) follows.

\section{Open problems}

(i) As we mentioned, we prove the attraction to $\mathcal{S}$, as stated in (3.17), but have not proved the attraction to a particular solitary wave like (2.4). It would be interesting to find solutions with multiple omega-limit points, that is, the situation when the frequency parameter $\omega$ keeps changing adiabatically.

(ii) Our argument does not apply to the Schrödinger equation. The important feature of the Klein-Gordon equation is that the continuous spectrum corresponds to $|\omega| \geq m$, hence the spectral density of the solution is absolutely continuous for $|\omega| \geq m$, while the spectrum of the omega-limit trajectory is within the compact set $[-m, m]$. This is not so for the Schrödinger equation: since the continuous spectrum corresponds to $\omega \geq 0$, the resulting restriction on the spectrum of the omega-limit trajectory is $\omega \leq 0$. As a result, we do not know whether the spectrum is compact; the Titchmarsh convolution theorem does not apply, and the proof breaks down. It would be extremely interesting to investigate whether the convergence to solitary waves is no longer true, or instead certain modification of the Titchmarsh theorem allows to reduce the spectrum to a point.

(iii) Similarly, the Titchmarsh theorem does not apply when the nonlinearity is not polynomial, and it would be interesting to investigate what could happen in such a case.

\section{Proof of attraction to solitary waves for the Klein-Gordon field with one nonlinear oscillator}

We will sketch the proof of Theorem 2 for the system (3.1) which describes one nonlinear oscillator located at the origin.

Proposition 2 (Compactness. Existence of omega-limit trajectories).

(i) For any sequence $s_{j} \rightarrow+\infty$ there exists a subsequence $s_{j^{\prime}} \rightarrow+\infty$ such that

$$
\psi\left(x, s_{j^{\prime}}+t\right) \rightarrow \beta(x, t), \quad x \in \mathbb{R}, \quad t \in \mathbb{R},
$$

for some $\beta \in C(\mathbb{R} \times \mathbb{R})$, where the convergence is in $C_{b}([-T, T] \times[-R, R])$, for any $T>0$ and $R>0$.

(ii)

$$
\sup _{t \in \mathbb{R}}\|\beta(\cdot, t)\|_{H^{1}}<\infty .
$$

Proof. By Theorem $1(v)$, for any $\epsilon \in[0,1]$,

$$
\psi \in C^{(\epsilon)}\left(\mathbb{R}, H^{1-\epsilon}(\mathbb{R})\right) .
$$


Taking $\epsilon=1 / 4$, we see that $\psi \in C^{(\alpha)}(\mathbb{R} \times \mathbb{R})$, for any $\alpha<1 / 4$. Now the first statement of the proposition follows by the Ascoli-Arzelà theorem. The bound (4.2) follows from (4.1), the bound (3.7), and the Fatou lemma.

We call omega-limit trajectory any function $\beta(x, t)$ that can appear as a limit in (4.1) (cf. Definition 3). We are going to prove that every omega-limit trajectory $\beta$ belongs to the set of solitary waves; that is,

$$
\beta(x, t)=\phi_{\omega_{+}}(x) e^{-i \omega_{+} t} \quad \text { for some } \quad \omega_{+} \in[-m, m] .
$$

Remark 6. The fact that any omega-limit trajectory turns out to be a solitary wave implies the following statement:

if there is a sequence $t_{j} \rightarrow \infty$ so that $\left(\psi\left(t_{j}\right), \dot{\psi}\left(t_{j}\right)\right) \stackrel{\mathscr{E}_{F}}{\longrightarrow} \Phi \in H^{1} \times L^{2}$, then $\Phi \in \mathcal{S}$. (4.4) In turn, (4.4) implies the convergence to the attractor in the metric (3.6) of $\mathscr{E}_{F}^{-\epsilon}$ for $\epsilon>0$ :

$$
(\psi(t), \dot{\psi}(t)) \stackrel{\mathscr{E}_{F}^{-\epsilon}}{\longrightarrow} \mathcal{S}, \quad \epsilon>0, \quad t \rightarrow \pm \infty
$$

This is weaker than the convergence to the attractor in the topology of $\mathscr{E}_{F}$ stated in Theorem 2. For the proof of the convergence to the attractor in the topology of $\mathscr{E}_{F}$, see [35].

Let us split the solution $\psi(x, t)$ into two components, $\psi(x, t)=\chi(x, t)+\varphi(x, t)$, which are defined for all $t \in \mathbb{R}$ as solutions to the following Cauchy problems:

$$
\begin{aligned}
& \ddot{\chi}(x, t)=\chi^{\prime \prime}(x, t)-m^{2} \chi(x, t),\left.\quad(\chi, \dot{\chi})\right|_{t=0}=\left(\psi_{0}(x), \pi_{0}(x)\right), \\
& \ddot{\varphi}(x, t)=\varphi^{\prime \prime}(x, t)-m^{2} \varphi(x, t)+\delta(x) f(t),\left.\quad(\varphi, \dot{\varphi})\right|_{t=0}=(0,0),
\end{aligned}
$$

where $\left(\psi_{0}(x), \pi_{0}(x)\right)$ is the initial data from (3.1), and

$$
f(t):=F(\psi(0, t)), \quad t \in \mathbb{R} .
$$

Lemma 1 (Local energy decay of the dispersive component). There is the following decay for $\chi$ :

$$
\lim _{t \rightarrow \infty}\|(\chi(\cdot, t), \dot{\chi}(\cdot, t))\|_{\mathscr{E}, R}=0, \quad \forall R>0 .
$$

For the proof, see [35, Lemma 3.1]. Lemma 1 means that the dispersive component $\chi$ does not give any contribution to the omega-limit trajectories (see Definition 3).

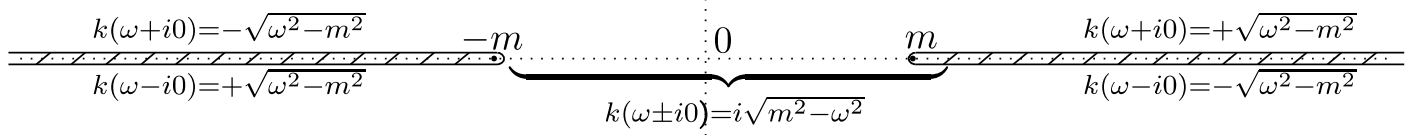

Figure 2. Domain $D$ and the values of $k(\omega \pm i 0), \omega \in \mathbb{R}$.

Let $k(\omega)$ be the analytic function with the domain $D:=\mathbb{C} \backslash((-\infty,-m] \cup[m,+\infty))$ such that

$$
k(\omega)=\sqrt{\omega^{2}-m^{2}}, \quad \operatorname{Im} k(\omega)>0, \quad \omega \in D .
$$


See Fig. 2. Let us also denote the limit of $k(\omega)$ for $\omega+i 0, \omega \in \mathbb{R}$, by

$$
k_{+}(\omega):=k(\omega+i 0), \quad \omega \in \mathbb{R} .
$$

The function $\varphi(x, t)=\psi(x, t)-\chi(x, t)$ satisfies the following Cauchy problem:

$$
\ddot{\varphi}(x, t)=\varphi^{\prime \prime}(x, t)-m^{2} \varphi(x, t)+\delta(x) f(t),\left.\quad(\varphi, \dot{\varphi})\right|_{t=0}=(0,0),
$$

with $f(t)$ defined in (4.6). Note that $\psi(0, \cdot) \in C_{b}(\mathbb{R})$ by the Sobolev embedding, since $(\psi, \dot{\psi}) \in$ $C_{b}(\mathbb{R}, \mathscr{E})$ by Theorem $1(i v)$. Hence, $f(t) \in C_{b}(\mathbb{R})$. On the other hand, since $\chi(x, t)$ is a finite energy solution to the free Klein-Gordon equation, $(\chi, \dot{\chi}) \in C_{b}(\mathbb{R}, \mathscr{E})$. It follows that $\varphi=\psi-\chi$ is also of finite energy norm:

$$
(\varphi, \dot{\varphi}) \in C_{b}(\mathbb{R}, \mathscr{E})
$$

We denote

$$
\varphi_{+}(x, t):=\theta(t) \varphi(x, t), \quad f_{+}(t):=\theta(t) f(t)=\theta(t)(\psi(0, t)) .
$$

The function $\varphi_{+}(x, t)$ satisfies the equation

$$
\ddot{\varphi}_{+}(x, t)=\partial_{x}^{2} \varphi_{+}(x, t)-m^{2} \varphi_{+}(x, t)+\delta(x) f_{+}(t),\left.\quad\left(\varphi_{+}, \dot{\varphi}_{+}\right)\right|_{t=0}=(0,0), \quad t \in \mathbb{R} .
$$

We set $\mathcal{F}_{t \rightarrow \omega}[g(t)](\omega)=\int_{\mathbb{R}} e^{i \omega t} g(t) d t$ for a function $g(t)$ from the Schwartz space $\mathscr{S}(\mathbb{R})$. The Fourier transform

$$
\hat{\varphi}_{+}(x, \omega)=\mathcal{F}_{t \rightarrow \omega}\left[\varphi_{+}(x, t)\right]=\int_{0}^{\infty} e^{i \omega t} \varphi(x, t) d t, \quad(x, \omega) \in \mathbb{R}^{2},
$$

is a continuous function of $x \in \mathbb{R}$ with values in tempered distributions of $\omega \in \mathbb{R}$, which satisfies the following equation (cf. (4.10)):

$$
-\omega^{2} \hat{\varphi}_{+}(x, \omega)=\partial_{x}^{2} \hat{\varphi}_{+}(x, \omega)-m^{2} \hat{\varphi}_{+}(x, \omega)+\delta(x) \hat{f}_{+}(\omega), \quad(x, \omega) \in \mathbb{R}^{2} .
$$

Proposition 3 (Spectral representation). There is the following relation:

$$
\hat{\varphi}_{+}(x, \omega)=\hat{\varphi}_{+}(0, \omega) e^{i k_{+}(\omega)|x|}, \quad x \in \mathbb{R} .
$$

Proof. Let us analyze the complex Fourier transform of $\varphi_{+}(x, t)$ :

$$
\tilde{\varphi}_{+}(x, \omega)=\mathcal{F}_{t \rightarrow \omega}\left[\varphi_{+}(x, t)\right]=\int_{0}^{\infty} e^{i \omega t} \varphi(x, t) d t, \quad x \in \mathbb{R}, \quad \omega \in \mathbb{C}^{+},
$$

where $\mathbb{C}^{+}:=\{z \in \mathbb{C}: \operatorname{Im} z>0\}$. Due to $(4.9), \tilde{\varphi}_{+}(\cdot, \omega)$ are $H^{1}$-valued analytic functions of $\omega \in \mathbb{C}^{+}$. Equation (4.10) implies that $\tilde{\varphi}_{+}$satisfies

$$
-\omega^{2} \tilde{\varphi}_{+}(x, \omega)=\partial_{x}^{2} \tilde{\varphi}_{+}(x, \omega)-m^{2} \tilde{\varphi}_{+}(x, \omega)+\delta(x) \tilde{f}_{+}(\omega), \quad \omega \in \mathbb{C}^{+} .
$$

The fundamental solutions $G_{ \pm}(x, \omega)=\frac{e^{ \pm i k(\omega)|x|}}{ \pm 2 i k(\omega)}$ satisfy

$$
G_{ \pm}^{\prime \prime}(x, \omega)+\left(\omega^{2}-m^{2}\right) G_{ \pm}(x, \omega)=\delta(x), \quad \omega \in \mathbb{C}^{+} .
$$

Note that for each $\omega \in \mathbb{C}^{+}$the function $G_{+}(\cdot, \omega)$ is in $H^{1}(\mathbb{R})$ by definition $(4.7)$, while $G_{-}(\cdot, \omega)$ is not. The solution $\tilde{\varphi}_{+}(x, \omega)$ can be written as a linear combination of these fundamental 
solutions. We use the standard "limiting absorption principle" for the selection of the appropriate fundamental solution: Since $\tilde{\varphi}_{+}(\cdot, \omega) \in H^{1}(\mathbb{R})$ for $\omega \in \mathbb{C}^{+}$, so is $G_{+}(\cdot, \omega)$, while $G_{-}(\cdot, \omega)$ is not, we have:

$$
\tilde{\varphi}_{+}(x, \omega)=-\tilde{f}_{+}(\omega) G_{+}(x, \omega)=-\tilde{f}_{+}(\omega) \frac{e^{i k(\omega)|x|}}{2 i k(\omega)}, \quad \omega \in \mathbb{C}^{+} .
$$

The relation (4.12) yields

$$
\tilde{\varphi}_{+}(x, \omega)=-\tilde{f}_{+}(\omega) \frac{e^{i k(\omega)|x|}}{2 i k(\omega)}=e^{i k(\omega)|x|} \tilde{\varphi}_{+}(0, \omega), \quad x \in \mathbb{R}, \quad \omega \in \mathbb{C}^{+} .
$$

Now we extend the relation (4.13) to $\omega \in \mathbb{R}$. Since $\varphi \in C_{b}\left(\mathbb{R}, H^{1}(\mathbb{R})\right)$ by (4.9), we have

$$
\theta(t) \varphi(x, t)=\lim _{\varepsilon \rightarrow 0+} \theta(t) \varphi(x, t) e^{-\varepsilon t}
$$

where the convergence holds in the space of $H^{1}$-valued tempered distributions, $\mathscr{S}^{\prime}\left(\mathbb{R}, H^{1}(\mathbb{R})\right)$. The Fourier transform $\hat{\varphi}_{+}(x, \omega):=\mathcal{F}_{t \rightarrow \omega}\left[\varphi_{+}(x, t)\right]=\mathcal{F}_{t \rightarrow \omega}[\theta(t) \varphi(x, t)]$ is defined as a tempered $H^{1}$-valued distribution of $\omega \in \mathbb{R}$. As follows from (4.14) and the continuity of the Fourier transform $\mathcal{F}_{t \rightarrow \omega}$ in $\mathscr{S}^{\prime}(\mathbb{R}), \hat{\varphi}_{+}(x, \omega)$ is the boundary value of the analytic function $\tilde{\varphi}_{+}(x, \omega)$, in the following sense:

$$
\hat{\varphi}_{+}(x, \omega)=\lim _{\varepsilon \rightarrow 0+} \tilde{\varphi}_{+}(x, \omega+i \varepsilon)=\lim _{\varepsilon \rightarrow 0+} \mathcal{F}_{t \rightarrow \omega}\left[\theta(t) \varphi(x, t) e^{-\varepsilon t}\right], \quad \omega \in \mathbb{R} .
$$

Again, the convergence is in the space $\mathscr{S}^{\prime}\left(\mathbb{R}, H^{1}(\mathbb{R})\right)$.

We use (4.15) to take the limit $\operatorname{Im} \omega \rightarrow 0+$ in the expression (4.13) for $\tilde{\varphi}_{+}(x, \omega)$, and keep in mind that $\tilde{\varphi}_{+}(x, \omega)$ is a quasimeasure (see Remark 7) for each $x \in \mathbb{R}$, while the exponential factor in (4.13) is a multiplicator in the space of quasimeasures. The formula (4.11) follows.

Remark 7. A tempered distribution $\mu(\omega) \in \mathscr{S}^{\prime}(\mathbb{R})$ is called a quasimeasure if $\check{\mu}(t)=\mathscr{F}_{\omega \rightarrow t}^{-1}[\mu(\omega)]$ $\in C_{b}(\mathbb{R})$. For more details on quasimeasures and multiplicators in the space of quasimeasures, see [35, Appendix B].

Proposition 4 (Absolute continuity of the spectrum). The distribution $\hat{\varphi}_{+}(0, \omega)$ is absolutely continuous for $|\omega|>m$, and moreover

$$
\int_{\mathbb{R} \backslash[-m, m]}\left|\hat{\varphi}_{+}(0, \omega)\right|^{2} \frac{k_{+}(\omega)}{\omega} d \omega<\infty
$$

where $k_{+}(\omega) / \omega>0$ for $\omega \in \mathbb{R} \backslash[-m, m]$ (see (4.8) and Fig. 2).

Proof. We use the Paley-Wiener arguments. Namely, the Parseval identity and (4.9) imply that

$$
\int_{\mathbb{R}}\left\|\tilde{\varphi}_{+}(\cdot, \omega+i \varepsilon)\right\|_{L^{2}}^{2} d \omega=2 \pi \int_{0}^{\infty} e^{-2 \varepsilon t}\left\|\varphi_{+}(\cdot, t)\right\|_{L^{2}}^{2} d t \leq \frac{C}{\varepsilon}, \quad \varepsilon>0 .
$$

On the other hand, we can calculate the term in the left-hand side of (4.17) exactly. According to $(4.13)$,

$$
\tilde{\varphi}_{+}(x, \omega+i \varepsilon)=\tilde{\varphi}_{+}(0, \omega+i \varepsilon) e^{i k(\omega+i \varepsilon)|x|},
$$

hence (4.17) results in

$$
\varepsilon \int_{\mathbb{R}}\left|\tilde{\varphi}_{+}(0, \omega+i \varepsilon)\right|^{2}\left\|e^{i k(\omega+i \varepsilon)|x|}\right\|_{L^{2}}^{2} d \omega \leq C, \quad \varepsilon>0 .
$$


Here is a crucial observation about the norm of $e^{i k(\omega+i \varepsilon)|x|}$.

\section{Lemma 2.}

(i) For $\omega \in \mathbb{R} \backslash(-m, m)$,

$$
\lim _{\varepsilon \rightarrow 0+} \varepsilon\left\|e^{i k(\omega+i \varepsilon)|x|}\right\|_{L^{2}}^{2}=\frac{k_{+}(\omega)}{\omega} .
$$

(ii) For any $\delta>0$ there exists $\varepsilon_{\delta}>0$ such that for $|\omega|>m+\delta$ and $\varepsilon \in\left(0, \varepsilon_{\delta}\right)$,

$$
\varepsilon\left\|e^{i k(\omega+i \varepsilon)|x|}\right\|_{L^{2}}^{2} \geq \frac{k_{+}(\omega)}{2 \omega} .
$$

Remark 8. The asymptotic behavior of the $L^{2}$-norm of $e^{i k(\omega+i \varepsilon)}$ stated in the lemma is easy to understand: for $\omega \in \mathbb{R} \backslash[-m, m]$, this norm is finite for $\varepsilon>0$ due to the small positive imaginary part of $k(\omega+i \varepsilon)$, but it becomes unboundedly large when $\varepsilon \rightarrow 0+$. Let us also mention that the integral (4.19) is easy to evaluate in the momentum space.

Substituting (4.20) into (4.18), we get:

$$
\int_{|\omega| \geq m+\delta}\left|\tilde{\varphi}_{+}(0, \omega+i \varepsilon)\right|^{2} \frac{k_{+}(\omega)}{\omega} d \omega \leq 2 C, \quad 0<\varepsilon<\varepsilon_{\delta},
$$

with the same $C$ as in (4.18). We conclude that for each $\delta>0$ the set of functions

$$
g_{\delta, \varepsilon}(\omega)=\tilde{\varphi}_{+}(0, \omega+i \varepsilon)\left|\frac{k_{+}(\omega)}{\omega}\right|^{1 / 2}, \quad \varepsilon \in\left(0, \varepsilon_{\delta}\right),
$$

defined for $\omega \in \Omega_{\delta}$, is bounded in the Hilbert space $L^{2}(\mathbb{R} \backslash[-m-\delta, m+\delta])$, and, by the Banach Theorem, is weakly compact. The convergence of the distributions (4.15) implies the following weak convergence in the Hilbert space $L^{2}(\mathbb{R} \backslash[-m-\delta, m+\delta])$ :

$$
g_{\delta, \varepsilon} \rightarrow g_{\delta}, \quad \varepsilon \rightarrow 0+
$$

where the limit function $g_{\delta}(\omega)$ coincides with the distribution $\hat{\varphi}_{+}(0, \omega)\left|\frac{k_{+}(\omega)}{\omega}\right|^{1 / 2}$ restricted onto $\mathbb{R} \backslash[-m-\delta, m+\delta]$. It remains to note that, by (4.21), the norms of all functions $g_{\delta}, \delta>0$, are bounded in $L^{2}(\mathbb{R} \backslash[-m-\delta, m+\delta])$ by a constant independent on $\delta$, hence (4.16) follows.

By Lemma 1, the dispersive component $\chi(\cdot, t)$ converges to zero in $\mathscr{E}_{F}$ as $t \rightarrow \infty$. On the other hand, by (4.1), $\psi\left(x, t+s_{j^{\prime}}\right)$ converges to $\beta(x, t)$ as $j^{\prime} \rightarrow \infty$, uniformly on every compact set of the plane $\mathbb{R}^{2}$. Hence, $\varphi\left(x, t+s_{j^{\prime}}\right)=\psi\left(x, t+s_{j^{\prime}}\right)-\chi\left(x, t+s_{j^{\prime}}\right)$ also converges to $\beta(x, t)$, uniformly in every compact set of the plane $\mathbb{R}^{2}$ :

$$
\varphi\left(x, s_{j^{\prime}}+t\right) \rightarrow \beta(x, t), \quad x \in \mathbb{R}, \quad t \in \mathbb{R} .
$$

Therefore, taking the limit in equation (4.5), we conclude that the omega-limit trajectory $\beta(x, t)$ also satisfies the same equation:

$$
\ddot{\beta}(x, t)=\beta^{\prime \prime}(x, t)-m^{2} \beta(x, t)+\delta(x) F(\beta), \quad x \in \mathbb{R}, \quad t \in \mathbb{R} .
$$

Taking the Fourier transform of $\beta$ in time, we see by $(4.1)$ that $\hat{\beta}(x, \omega)$ is a continuous function of $x \in \mathbb{R}$, with values in tempered distributions of $\omega \in \mathbb{R}$, and that it satisfies the corresponding stationary equation

$$
-\omega^{2} \hat{\beta}(x, \omega)=\hat{\beta}^{\prime \prime}(x, \omega)-m^{2} \hat{\beta}(x, \omega)+\delta(x) \hat{g}(\omega), \quad(x, \omega) \in \mathbb{R}^{2},
$$


valid in the sense of tempered distributions of $(x, \omega) \in \mathbb{R}^{2}$, where $\hat{g}(\omega)$ are the Fourier transforms of the function

$$
g(t):=F(\beta(0, t))
$$

For brevity, we denote

$$
\beta(t):=\beta(0, t) .
$$

\section{Lemma 3 (Boundedness of spectrum).}

$$
\operatorname{supp} \hat{\beta} \subset[-m, m] \text {. }
$$

Proof. By (4.22), we have

$$
\varphi_{+}\left(x, s_{j^{\prime}}+t\right) \rightarrow \beta(x, t), \quad x \in \mathbb{R}, \quad t \in \mathbb{R},
$$

with the same convergence as in (4.1) and (4.22). We have:

$$
\varphi_{+}\left(x, s_{j}+t\right)=\frac{1}{2 \pi} \int_{\mathbb{R}} e^{-i \omega t} e^{-i \omega s_{j}} \hat{\varphi}_{+}(x, \omega) d \omega, \quad x \in \mathbb{R}, \quad t \in \mathbb{R},
$$

where the integral is understood as the pairing of a smooth function (oscillating exponent) with a compactly supported distribution. Hence, (4.24) implies that

$$
e^{-i \omega s_{j^{\prime}}} \hat{\varphi}_{+}(x, \omega) \rightarrow \hat{\beta}(x, \omega), \quad x \in \mathbb{R}, \quad s_{j^{\prime}} \rightarrow \infty,
$$

in the sense of quasimeasures (the convergence in the space of quasimeasures is equivalent to the Ascoli-Arzelà type convergence of corresponding Fourier transforms; see [35, Appendix B]). Since $\hat{\varphi}_{+}(0, \omega)$ is locally $L^{2}$ for $|\omega|>m$ by Proposition 4 , the convergence (4.25) at $x=0$ shows that $\hat{\beta}(\omega):=\hat{\beta}(0, \omega)$ vanishes for $|\omega|>m$. This proves the lemma.

We denote

$$
\kappa(\omega):=-i k_{+}(\omega), \quad \omega \in \mathbb{R},
$$

where $k_{+}(\omega)$ was introduced in (4.8). We then have $\operatorname{Re} \kappa(\omega) \geq 0$, and also

$$
\kappa(\omega)=\sqrt{\omega^{2}-m^{2}}>0 \quad \text { for } \quad-m<\omega<m,
$$

in accordance with (3.10).

Proposition 5 (Spectral representation for $\beta$ ). The distribution $\hat{\beta}(x, \omega)$ admits the following representation:

$$
\hat{\beta}(x, \omega)=\hat{\beta}(\omega) e^{-\kappa(\omega)|x|}, \quad x \in \mathbb{R} .
$$

Proof. This follows by taking the limit in the first line of (4.11), since supp $\hat{\beta} \subset[-m, m]$ by Lemma 3, while $k(\omega)=i \kappa(\omega)$ for $-m \leq \omega \leq m$ (cf. (4.26)).

Proposition 6 (Reduction to point spectrum). Either $\operatorname{supp} \hat{\beta}=\left\{\omega_{+}\right\}$for some $\omega_{+} \in$ $[-m, m]$ or $\hat{\beta}=0$. 
Proof. By Lemma 3, we know that supp $\hat{\beta} \subset[-m, m]$. According to equation (4.23), the function $\hat{\beta}$ satisfies the following jump condition at the point $x=0$ :

$$
\hat{\beta}^{\prime}(0+, \omega)-\hat{\beta}^{\prime}(0-, \omega)=\hat{g}(\omega), \quad \omega \in \mathbb{R} .
$$

Since $\operatorname{supp} \hat{\beta}^{\prime}(0 \pm, \cdot) \subset \operatorname{supp} \hat{\beta}$ by Proposition 5 , it follows that

$$
\operatorname{supp} \hat{g}(\cdot) \subset \operatorname{supp} \hat{\beta} \text {. }
$$

On the other hand, by (3.13), the Fourier transform $\hat{g}(\omega)$ of $g(t):=F(\beta(0, t))$ is given by

$$
\hat{g}=-\sum_{n=1}^{p} 2 n u_{n} \underbrace{(\hat{\beta} * \hat{\bar{\beta}}) * \cdots *(\hat{\beta} * \hat{\bar{\beta}})}_{n-1} * \hat{\beta} .
$$

Now we will use the Titchmarsh convolution theorem [57] (see also [38, p. 119] and [24, Theorem 4.3.3]) which could be stated as follows:

For any compactly supported distributions $u$ and $v, \sup \operatorname{supp}(u * v)=\sup \operatorname{supp} u+\sup \operatorname{supp} v$.

Applying the Titchmarsh convolution theorem to the convolutions in (4.28), we obtain the following equality:

$$
\sup \operatorname{supp} \hat{g} \geq \sup \operatorname{supp} \hat{\beta}+(p-1)(\sup \operatorname{supp} \hat{\beta}-\inf \operatorname{supp} \hat{\beta})
$$

where we used the relation sup supp $\hat{\bar{\beta}}=-\inf \operatorname{supp} \hat{\beta}$. We wrote " $\geq$ " because of possible cancellations in the summation in the right-hand side of (4.28). Note that the Titchmarsh theorem is applicable to (4.28) since supp $\hat{\beta}$ is compact by Lemma 3 .

Comparing (4.27) with (4.29), we conclude that

$$
(p-1)(\sup \operatorname{supp} \hat{\beta}-\inf \operatorname{supp} \hat{\beta})=0 \text {. }
$$

Since $p \geq 2$ by (3.13) (which means that the oscillator at $x=0$ is nonlinear), we conclude that $\operatorname{supp} \hat{\beta}$ consists of at most a single point $\omega_{+} \subset[-m, m]$.

By Proposition 6, supp $\hat{\beta} \subset\left\{\omega_{+}\right\}$, with $\omega_{+} \in[-m, m]$. Therefore,

$$
\hat{\beta}(\omega)=a_{1} \delta\left(\omega-\omega_{+}\right), \quad \text { with some } a_{1} \in \mathbb{C} \text {. }
$$

Note that the derivatives $\delta^{(k)}\left(\omega-\omega_{+}\right), k \geq 1$ do not enter the expression for $\hat{\beta}(\omega)$ since $\beta(t)=$ $\beta(0, t)$ is a bounded continuous function of $t$ due to the bound (4.2). Proposition 5 and (4.30) imply that the omega-limit trajectory $\beta(x, t)$ is a solitary wave:

$$
\beta(x, t)=\phi(x) e^{-i \omega_{+} t},
$$

where $\phi \in H^{1}(\mathbb{R})$ by (4.2). This completes the proof of (4.3).

Remark 9. $\omega_{+}= \pm m$ could only correspond to the zero solution by Remark 5 . 


\section{Multifrequency solitons}

\subsection{Linear degeneration}

Let us consider equation (3.3) with $N=2$, under condition (3.14).

Proposition 7. If in (3.13) one has $p_{J}=1$ for some $J$, then the conclusion of Theorem 2 may no longer be correct.

Proof. We are going to construct the multifrequency solitary waves. Consider the equation

$$
\ddot{\psi}=\psi^{\prime \prime}-m^{2} \psi+\delta(x) F_{1}(\psi)+\delta(x-L) F_{2}(\psi),
$$

where

$$
F_{1}(\psi)=\alpha \psi+\beta|\psi|^{2} \psi, \quad F_{2}(\psi)=\gamma \psi, \quad \alpha, \beta, \gamma \in \mathbb{R} .
$$

Note that the function $F_{2}$ is linear, failing to satisfy (3.13) (where one now has $p_{2}=1$ ). The function

$$
\psi(x, t)=\left\{\begin{array}{l}
(A+B) e^{\kappa(\omega) x} \sin \omega t, \quad x \leq 0, \\
\left(A e^{-\kappa(\omega) x}+B e^{\kappa(\omega) x}\right) \sin \omega t+C \sinh (\kappa(3 \omega) x) \sin 3 \omega t, \quad x \in[0, L], \\
\left(A e^{-\kappa(\omega)}+B e^{\kappa(\omega)(2 L-x)}\right) \sin \omega t+\frac{C}{\sinh (\kappa(3 \omega) L)} e^{-\kappa(3 \omega)(x-L)} \sin 3 \omega t, \quad x \geq L,
\end{array}\right.
$$

where $\omega \in(0, m / 3)$, will be a solution if the jump conditions are satisfied at $x=0$ and at $x=L$ :

$$
\begin{aligned}
& -\psi^{\prime}(0+, t)+\psi^{\prime}(0-, t)=\alpha \psi(0, t)+\beta \psi^{3}(0, t), \\
& -\psi^{\prime}(L+, t)+\psi^{\prime}(L-, t)=\alpha \psi(L, t)+\beta \psi^{3}(L, t) .
\end{aligned}
$$

Using the identity

$$
\sin ^{3} \theta=\frac{3}{4} \sin \theta-\frac{1}{4} \sin 3 \theta
$$

we see that

$$
\begin{aligned}
\alpha(A+B) \sin \omega t+\beta((A+B) \sin \omega t)^{3} \\
\quad=\left(\alpha(A+B)+\beta \frac{3(A+B)^{3}}{4}\right) \sin \omega t-\beta \frac{(A+B)^{3}}{4} \sin 3 \omega t .
\end{aligned}
$$

Collecting the terms at $\sin \omega t$ and at $\sin 3 \omega t$, we write the condition (5.1) as the following system of equations:

$$
\begin{aligned}
& 2 \kappa(\omega) A=\left(\alpha(A+B)+\beta \frac{3(A+B)^{3}}{4}\right), \\
& -\kappa(3 \omega) C=-\beta \frac{(A+B)^{3}}{4} .
\end{aligned}
$$

Similarly, the condition (5.2) is equivalent to the following two equations:

$$
\begin{aligned}
& 2 B \kappa(\omega) e^{\kappa(\omega) L}=\gamma\left(A e^{-\kappa(\omega) L}+B e^{\kappa(\omega) L}\right), \\
& \frac{\kappa(3 \omega) C}{\sinh (\kappa(3 \omega) L)}+\kappa(3 \omega) C \cosh (\kappa(3 \omega) L)=\gamma C \sinh (\kappa(3 \omega) L) .
\end{aligned}
$$

Equations (5.4), (5.5), (5.6), and (5.7) could be satisfied for arbitrary $L>0$. Namely, for any $\omega \in(0, m / 3)$, one uses (5.7) to determine $\gamma$. For any $\beta \neq 0$, there is always a solution $A$, and $B$ to the nonlinear system (5.4), (5.6). Finally, $C$ is obtained from (5.5). 


\subsection{Wide gaps}

Let us consider equation (3.3) with $N=2$. Assume that (3.13) is satisfied.

Proposition 8. If the condition (3.14) is violated, then the conclusion of Theorem 2 may no longer be correct.

Proof. We will show that if $L:=X_{2}-X_{1}$ is sufficiently large, then one can take $F_{1}(\psi)$ and $F_{2}(\psi)$ satisfying (3.13) such that the global attractor of the equation contains the multifrequency solutions which do not converge to solitary waves of the form (3.8). For our convenience, we assume that $X_{1}=0, X_{2}=L$. We consider the model (3.3) with

$$
F_{1}(\psi)=F_{2}(\psi)=F(\psi), \quad \text { where } \quad F(\psi)=\alpha \psi+\beta|\psi|^{2} \psi, \quad \alpha, \beta \in \mathbb{R} .
$$

In terms of the condition (3.13), $p_{1}=p_{2}=2$. We take $L$ to be large enough:

$$
L>\frac{\pi}{2^{3 / 2} m} .
$$

Consider the function

$$
\psi(x, t)=A\left(e^{-\kappa(\omega)|x|}+e^{-\kappa(\omega)|x-L|}\right) \sin \omega t+B \chi_{[0, L]}(x) \sin (k(3 \omega) x) \sin 3 \omega t, \quad A, B \in \mathbb{C} .
$$

Then $\psi(x, t)$ solves (3.3) for $x$ away from the points $X_{J}$. We require that

$$
k(3 \omega)=\frac{\pi}{L},
$$

so that $\psi(x, t)$ is continuous in $x \in \mathbb{R}$ and symmetric with respect to $x=L / 2$ :

$$
\psi(x, t)=\psi\left(\frac{L}{2}-x, t\right), \quad x \in \mathbb{R} .
$$

We need $|\omega|<m$ to have $\kappa(\omega)>0$, and $3|\omega|>m$ to have $k(3 \omega) \in \mathbb{R}$. We take $\omega>0$, and thus $m<3 \omega<3 m$. By (5.9), this means that we need

$$
m<\sqrt{\frac{\pi^{2}}{L^{2}}+m^{2}}<3 m
$$

The second inequality is satisfied by (5.8).

Due to the symmetry of $\psi(x, t)$ with respect to $x=L / 2$, the jump condition both at $x=$ $X_{1}=0$ and at $x=X_{2}=L$ takes the following identical form:

$$
2 A \kappa(\omega) \sin \omega t-B k(3 \omega) \sin 3 \omega t=F\left(A\left(1+e^{-\kappa(\omega) L}\right) \sin \omega t\right) .
$$

We use the following relation which follows from (5.3):

$$
\begin{aligned}
F\left(A\left(1+e^{-\kappa(\omega) L}\right) \sin \omega t\right)= & \left(\alpha A\left(1+e^{-\kappa(\omega) L}\right)+\frac{3}{4} \beta|A|^{2} A\left(1+e^{-\kappa(\omega) L}\right)^{3}\right) \sin \omega t \\
& -\frac{1}{4} \beta|A|^{2} A\left(1+e^{-\kappa(\omega) L}\right)^{3} \sin 3 \omega t .
\end{aligned}
$$

Collecting in (5.10) the terms at $\sin \omega t$ and at $\sin 3 \omega t$, we obtain the following system:

$$
\begin{aligned}
& 2 A \kappa(\omega)=\alpha A\left(1+e^{-\kappa(\omega) L}\right)+\frac{3}{4} \beta|A|^{2} A\left(1+e^{-\kappa(\omega) L}\right)^{3}, \\
& B k(3 \omega)=\frac{1}{4} \beta|A|^{2} A\left(1+e^{-\kappa(\omega) L}\right)^{3} .
\end{aligned}
$$


Assuming that $A \neq 0$, we divide the first equation by $A$ :

$$
2 \kappa(\omega)=\alpha\left(1+e^{-\kappa(\omega) L}\right)+\frac{3}{4} \beta|A|^{2}\left(1+e^{-\kappa(\omega) L}\right)^{3} .
$$

The condition for the existence of a solution $A \neq 0$ is

$$
\left(\frac{2 \kappa(\omega)}{1+e^{-\kappa(\omega) L}}-\alpha\right) \beta>0 .
$$

Once we found $A$, the second equation in (5.11) can be used to express $B$ in terms of $A$.

Remark 10. Condition (5.12) shows that we can choose $\beta<0$ taking large $\alpha>0$. The corresponding potential $U(\psi)=-\alpha|\psi|^{2} / 2-\beta|\psi|^{4} / 4$ satisfies (3.13).

\section{References}

[1] Babin A.V., Vishik M.I., Attractors of evolution equations, of Studies in Mathematics and its Applications, Vol. 25, North-Holland Publishing Co., Amsterdam, 1992 (translated and revised from the 1989 Russian original by Babin).

[2] Berestycki H., Lions P.-L., Nonlinear scalar field equations. I. Existence of a ground state, Arch. Ration. Mech. Anal. 82 (1983), 313-345.

[3] Berestycki H., Lions P.-L., Nonlinear scalar field equations. II. Existence of infinitely many solutions, Arch. Ration. Mech. Anal. 82 (1983), 347-375.

[4] Bohr N., On the constitution of atoms and molecules, Phil. Mag. 26 (1913), 1-25.

[5] Brezis H., Lieb E.H., Minimum action solutions of some vector field equations, Comm. Math. Phys. 96 (1984), 97-113.

[6] Broglie L.D., Recherches sur la théorie des Quanta, Thèses, Paris, 1924.

[7] Buslaev V.S., Perel'man G.S., Scattering for the nonlinear Schrödinger equation: states that are close to a soliton, St. Petersburg Math. J. 4 (1993), 1111-1142.

[8] Buslaev V.S., Perel'man G.S., On the stability of solitary waves for nonlinear Schrödinger equations, in Nonlinear Evolution Equations, Amer. Math. Soc. Transl. Ser. 2, Vol. 164, Amer. Math. Soc., Providence, RI, 1995, 75-98.

[9] Buslaev V.S., Sulem C., On asymptotic stability of solitary waves for nonlinear Schrödinger equations, Ann. Inst. H. Poincaré Anal. Non Linéaire 20 (2003), 419-475.

[10] Cazenave T., Vázquez L., Existence of localized solutions for a classical nonlinear Dirac field, Comm. Math. Phys. 105 (1986), 35-47.

[11] Comech A.A., Numerical simulations of the Klein-Gordon field with nonlinear interaction (2007), scripts for GNU Octave, http://www.math.tamu.edu/ comech/tools/kg-string.

[12] Cuccagna S., Asymptotic stability of the ground states of the nonlinear Schrödinger equation, Rend. Istit. Mat. Univ. Trieste 32 (2001), 105-118.

[13] Cuccagna S., Stabilization of solutions to nonlinear Schrödinger equations, Comm. Pure Appl. Math. 54 (2001), 1110-1145.

[14] Cuccagna S., On asymptotic stability of ground states of NLS, Rev. Math. Phys. 15 (2003), 877-903.

[15] Derrick G.H., Comments on nonlinear wave equations as models for elementary particles, J. Math. Phys. 5 (1964), 1252-1254.

[16] Esteban M.J., Georgiev V., Séré E., Stationary solutions of the Maxwell-Dirac and the Klein-Gordon-Dirac equations, Calc. Var. Partial Differential Equations 4 (1996), 265-281.

[17] Esteban M.J., Séré É., Stationary states of the nonlinear Dirac equation: a variational approach, Comm. Math. Phys. 171 (1995), 323-350.

[18] Gell-Mann M., Ne'eman Y., The eightfold way, W.A. Benjamin, Inc., New York, NY, 1964.

[19] Ginibre J., Velo G., Time decay of finite energy solutions of the nonlinear Klein-Gordon and Schrödinger equations, Ann. Inst. H. Poincaré Phys. Théor. 43 (1985), 399-442. 
[20] Glassey R.T., Strauss W.A., Decay of a Yang-Mills field coupled to a scalar field, Comm. Math. Phys. 67 (1979), 51-67.

[21] Grillakis M., Shatah J., Strauss W., Stability theory of solitary waves in the presence of symmetry. I, J. Funct. Anal. 74 (1987), 160-197.

[22] Guo Y., Nakamitsu K., Strauss W., Global finite-energy solutions of the Maxwell-Schrödinger system, Comm. Math. Phys. 170 (1995), 181-196.

[23] Henry D., Geometric theory of semilinear parabolic equations, Springer, 1981.

[24] Hörmander L., The analysis of linear partial differential operators. I, 2nd ed., Springer Study Edition, Springer-Verlag, Berlin, 1990.

[25] Hörmander L., On the fully nonlinear Cauchy problem with small data. II, in Microlocal Analysis and Nonlinear Waves (Minneapolis, MN, 1988-1989), IMA Vol. Math. Appl., Vol. 30, Springer, New York, 1991, 51-81.

[26] Jörgens K., Das Anfangswertproblem im Grossen für eine Klasse nichtlinearer Wellengleichungen, Math. Z. 77 (1961), 295-308.

[27] Klainerman S., Long-time behavior of solutions to nonlinear evolution equations, Arch. Ration. Mech. Anal. 78 (1982), 73-98.

[28] Komech A., On transitions to stationary states in one-dimensional nonlinear wave equations, Arch. Ration. Mech. Anal. 149 (1999), 213-228.

[29] Komech A., Spohn H., Long-time asymptotics for the coupled Maxwell-Lorentz equations, Comm. Partial Differential Equations 25 (2000), 559-584.

[30] Komech A., Spohn H., Kunze M., Long-time asymptotics for a classical particle interacting with a scalar wave field, Comm. Partial Differential Equations 22 (1997), 307-335.

[31] Komech A., Vainberg B., On asymptotic stability of stationary solutions to nonlinear wave and Klein-Gordon equations, Arch. Ration. Mech. Anal. 134 (1996), 227-248.

[32] Komech A.I., Stabilization of the interaction of a string with a nonlinear oscillator, Mosc. Univ. Math. Bull. 46 (1991), 34-39.

[33] Komech A.I., On stabilization of string-nonlinear oscillator interaction, J. Math. Anal. Appl. 196 (1995), 384-409.

[34] Komech A.I., On attractor of a singular nonlinear U(1)-invariant Klein-Gordon equation, in Progress in analysis, Vol. I, II (2001, Berlin), World Sci. Publishing, River Edge, NJ, 2003, 599-611.

[35] Komech A.I., Komech A.A., Global attractor for a nonlinear oscillator coupled to the Klein-Gordon field, Arch. Ration. Mech. Anal. 185 (2007), 105-142, math.AP/0609013.

[36] Komech A.I., Komech A.A., On global attraction to quantum stationary states II. Several nonlinear oscillators coupled to massive scalar field, MPI Preprint Series 17/2007 (2007), available at http://www.mis.mpg.de/preprints/2007/prepr2007_17.html.

[37] Komech A.I., Komech A.A., On global attraction to quantum stationary states III. KleinGordon equation with mean field interaction, MPI Preprint Series 66/2007 (2007), available at http://www.mis.mpg.de/preprints/2007/prepr2007_66.html.

[38] Levin B.Y., Lectures on entire functions, Translations of Mathematical Monographs, Vol. 150, American Mathematical Society, Providence, RI, 1996 (in collaboration with and with a preface by Yu. Lyubarskii, M. Sodin and V. Tkachenko, translated from the Russian manuscript by Tkachenko).

[39] Morawetz C.S., Strauss W.A., Decay and scattering of solutions of a nonlinear relativistic wave equation, Comm. Pure Appl. Math. 25 (1972), 1-31.

[40] Pillet C.-A., Wayne C.E., Invariant manifolds for a class of dispersive, Hamiltonian, partial differential equations, J. Differential Equations 141 (1997), 310-326.

[41] Schiff L.I., Nonlinear meson theory of nuclear forces. I. Neutral scalar mesons with point-contact repulsion, Phys. Rev. 84 (1951), 1-9.

[42] Schiff L.I., Nonlinear meson theory of nuclear forces. II. Nonlinearity in the meson-nucleon coupling, Phys. Rev. 84 (1951), 10-11.

[43] Schrödinger E., Quantisierung als eigenwertproblem, Ann. Phys. 81 (1926), 109-139.

[44] Segal I.E., The global Cauchy problem for a relativistic scalar field with power interaction, Bull. Soc. Math. France 91 (1963), 129-135. 
[45] Segal I.E., Non-linear semi-groups, Ann. of Math. (2) 78 (1963), 339-364.

[46] Segal I.E., Quantization and dispersion for nonlinear relativistic equations, in Proc. Conf. "Mathematical Theory of Elementary Particles" (1965, Dedham, Mass.), M.I.T. Press, Cambridge, Mass., 1966, 79-108.

[47] Shatah J., Stable standing waves of nonlinear Klein-Gordon equations, Comm. Math. Phys. 91 (1983), 313-327.

[48] Shatah J., Unstable ground state of nonlinear Klein-Gordon equations, Trans. Amer. Math. Soc. 290 (1985), 701-710.

[49] Shatah J., Strauss W., Instability of nonlinear bound states, Comm. Math. Phys. 100 (1985), $173-190$.

[50] Soffer A., Weinstein M.I., Multichannel nonlinear scattering for nonintegrable equations, Comm. Math. Phys. 133 (1990), 119-146.

[51] Soffer A., Weinstein M.I., Multichannel nonlinear scattering for nonintegrable equations. II. The case of anisotropic potentials and data, J. Differential Equations 98 (1992), 376-390.

[52] Soffer A., Weinstein M.I., Resonances, radiation damping and instability in Hamiltonian nonlinear wave equations, Invent. Math. 136 (1999), 9-74, chao-dyn/9807003.

[53] Strauss W.A., Decay and asymptotics for $\square u=f(u)$, J. Funct. Anal. 2 (1968), 409-457.

[54] Strauss W.A., Existence of solitary waves in higher dimensions, Comm. Math. Phys. 55 (1977), $149-162$.

[55] Tao T., A (concentration-)compact attractor for high-dimensional non-linear Schrödinger equations, Dyn. Partial Differ. Equ. 4 (2007), 1-53, math.AP/0611402.

[56] Temam R., Infinite-dimensional dynamical systems in mechanics and physics, 2nd ed., Applied Mathematical Sciences, Vol. 68, Springer-Verlag, New York, 1997.

[57] Titchmarsh E., The zeros of certain integral functions, Proc. London Math. Soc. 25 (1926), 283-302.

[58] Vakhitov M.G., Kolokolov A.A., Stationary solutions of the wave equation in the medium with nonlinearity saturation, Radiophys. Quantum Electron. 16 (1973), 783-789. 